\title{
Oncogenic Fli-1 is a potential prognostic marker for the progression of epithelial ovarian cancer
}

\author{
Wei Song ${ }^{1 \dagger}$, Lingyun $\mathrm{Hu}^{2+}$, Wei $\mathrm{Li}^{1}$, Guanjun Wang ${ }^{1}$, Yan $\mathrm{Li}^{1}$, Lei Yan ${ }^{1}$, Ailing $\mathrm{Li}^{3^{*}}$ and Jiuwei Cui ${ }^{{ }^{*}}$
}

\begin{abstract}
Background: Ovarian cancer is the most lethal gynecologic malignancy, but its etiology remains poorly understood. This study investigated the role of Fli-1 in ovarian carcinogenesis and disease survival.

Methods: Fli-1 protein expression was evaluated by immunohistochemistry in 104 primary epithelial ovarian cancer (EOC) patients with known follow-up data and 20 controls. Correlation between Fli- 1 expression and clinical characteristics was evaluated with the logistic regression. Kaplan Meier analysis was used to assess the impact of Fli-1 expression on overall survival (OS) and disease-free survival (DFS). Cell proliferation and migration assay were used to explore the function of Fli-1 in ovarian cancer cells.

Results: Fli-1 was expressed in $74 \%$ cases and up-regulated in EOC tissues compared with normal control tissues $(p<0.05)$. The high expression of Fli-1 was significantly associated with advanced tumor stage, positive lymph nodal involvement, and poor OS and DFS ( $p<0.05)$. Further analysis showed Fli-1 is an independent prognostic factor for OS and DFS. Down-regulation of Fli-1 inhibited cell proliferation but did not affect cell migration in SKOV3 cells.

Conclusions: This study revealed that Fli-1 played an essential role in the development and progression of ovarian cancers. Its overexpression is intimately related to malignant phenotypes and poor clinical outcome, suggesting that Fli-1 is a potential prognostic marker and therapeutic molecular target in ovarian cancer.
\end{abstract}

Keywords: Epithelial ovarian cancer (EOC), Fli-1, Tumor stage, Overall survival

\section{Background}

Ovarian cancer is the leading cause of death from gynecologic malignancy in developed countries and the second leading cause in developing countries [1,2]. Epithelial ovarian cancer (EOC) accounts for $90 \%$ of ovarian cancers; however, its aetiology remains unknown. The origin and pathogenesis of EOC have been investigated but still poorly understood. Over the past decades, prognosis for patients with EOC has improved little,with $70-80 \%$ of the cases having a recurrence of the cancer and ultimately succumbing to the disease [3]. There are a number of genetic and epigenetic changes that lead to transformation of ovarian epithelial cells into tumor cells [4]. Recognizing the importance of molecular mechanism, it is urgent to

\footnotetext{
*Correspondence: liailing@hotmail.com; cuijiuwei@yahoo.com

${ }^{\dagger}$ Equal contributors

${ }^{3}$ Institute of Basic Medical Sciences, National Center of Biomedical Analysis,

27 Tai-Ping Road, Beijing 100850, China

${ }^{1}$ Cancer center, the First Hospital of Jilin University, 71 Xinmin Street,

Changchun 130021, China

Full list of author information is available at the end of the article
}

identify key molecular regulators in tumorigenesis to improve the prognosis assessment and treatment of EOC patients.

Friend leukemia virus integration 1 (Fli-1), a member of the ETS transcription factor family, is the target of insertional activation by Friend murine leukemia virus (F-MuLV) and is preferentially expressed in vascular endothelial cells and hematopoietic tissues [5,6]. Transcription factors of the ETS family regulate the expression of oncogenes, tumor suppressor genes, and some related genes of the vessel's formation, invasion and metastasis, and often correlate with poor survival in some types of cancers [7-10]. Fli-1 plays a critical role in normal development, hematopoiesis and oncogenesis by functioning as both transcriptional activator and repressor [11-15]. Knocking-down Fli-1 expression in erythroleukemic cells leads to a marked growth inhibition and cell death, demonstrating a possible therapeutic approach to induce tumor suppression [16-19].

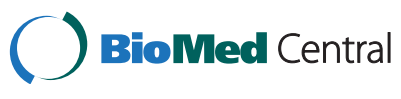

(c) 2014 Song et al.; licensee BioMed Central Ltd. This is an Open Access article distributed under the terms of the Creative Commons Attribution License (http://creativecommons.org/licenses/by/2.0), which permits unrestricted use, distribution, and reproduction in any medium, provided the original work is properly credited. The Creative Commons Public Domain Dedication waiver (http://creativecommons.org/publicdomain/zero/1.0/) applies to the data made available in this article, unless otherwise stated. 
It has also been shown that Fli-1 maintains several malignant phenotypes by inhibiting Rb, GATA-1, SHIP1 and targeting Bcl-2 [20-22]. Anti-Fli-1 compounds had been discovered and demonstrated strong anti-leukemic activity in a mouse erythroleukemia model that overexpresses Fli-1, making it possible for targeting Fli-1 as a potent treatment strategy [23].

However, the role of Fli-1 in EOC remains unknown. Here, we analyzed Fli-1 expression in EOC patients and studied its function in an ovary cell line.

\section{Methods}

\section{Patients and samples}

Formalin-fixed paraffin-embedded tissues of ovary and fallopian tube from primary ovarian cancer patients and control group, such as uterine prolapse, uterine fibroid were obtained from the First Hospital of Jilin University and the General Hospital of Chinese People's Liberation Army between 2005 and June 2009. The specimens included 104 Ovarian Serous Carcinoma, 10 fallopian tube and 10 normal ovaries. Primitive neuroectodermal tumor (PNET) was chosen as the positive controls at the same time. Clinical stage, histological grade and follow-up data were available for the majority of these patients. The histological subtypes and disease stages of the tumors were classified according to International Federation of Gynecology and Obstetrics (FIGO) criteria. Approval for the study was obtained from the Research Ethics Board of the First Hospital of Jilin University and the General Hospital of Chinese People's Liberation Army. The study participants gave their written informed consent. The clinical characteristics of all patients and the control group were shown in Table 1. The patients were followed up for survival analysis.

\section{Immunohistochemistry}

Tissue slides were de-paraffinized with xylene and rehydrated through a gradual decline of alcohol (100-80\%), and then incubated in 3\% hydrogen peroxide for 15 minutes to block endogenous peroxidase activity. Antigen retrieval was carried out by immersing the slides in $10 \mathrm{mM}$ sodium citrate buffer ( $\mathrm{pH}$ 6.0) and maintained at a sub-boiling temperature for 15 minutes. The slides were rinsed in phosphate-buffered saline and incubated with $10 \%$ normal goat serum to block non-specific staining for 30 minutes at $37^{\circ} \mathrm{C}$. Primary anti-Fli-1 polyclonal antibodies (Neomarker) were diluted in 1:100, and incubated with the sections at $4^{\circ} \mathrm{C}$ overnight. After washing with PBS, the secondary antibodies (biotinylated goat anti-rabbit immunoglobulin) and streptavidin peroxidase complex reagent were applied. Subsequently, the visualization signal was processed according to the Polink-2 HRP DAB Detection kit. Finally, the slides were counterstained with hematoxylin for $15 \mathrm{~min}$ and
Table 1 Clinicopathologic characteristics of EOC patients

\begin{tabular}{|c|c|c|}
\hline \multirow{2}{*}{$\begin{array}{l}\text { Variable } \\
\text { Age median (range) }\end{array}$} & \multicolumn{2}{|c|}{ Total number \% } \\
\hline & 52 & \\
\hline$<50$ & 43 & 41 \\
\hline$\geq 50$ & 61 & 59 \\
\hline \multicolumn{3}{|l|}{ FIGO stage } \\
\hline I & 20 & 19 \\
\hline$\|$ & 16 & 16 \\
\hline III & 50 & 48 \\
\hline IV & 18 & 17 \\
\hline \multicolumn{3}{|l|}{ Histological grade } \\
\hline G1 & 10 & 10 \\
\hline G2 & 14 & 13 \\
\hline G3 & 80 & 77 \\
\hline \multicolumn{3}{|l|}{ Lymph nodal involvement } \\
\hline Positive & 40 & 38 \\
\hline Negative & 64 & 62 \\
\hline \multicolumn{3}{|l|}{ Residual tumor size } \\
\hline $0 \mathrm{~mm}$ & 76 & 75 \\
\hline 0-10 mm & 28 & 25 \\
\hline CA125 serum level median (range) & 263 & \\
\hline$\leq 35 \mathrm{U} / \mathrm{ml}$ & 7 & 7 \\
\hline$>35 \mathrm{U} / \mathrm{ml}$ & 97 & 93 \\
\hline \multicolumn{3}{|l|}{ ER expression } \\
\hline Positive & 58 & 56 \\
\hline Negative & 46 & 44 \\
\hline \multicolumn{3}{|l|}{ PR expression } \\
\hline Positive & 47 & 45 \\
\hline Negative & 57 & 55 \\
\hline \multicolumn{3}{|l|}{ Her1 expression } \\
\hline Positive & 37 & 36 \\
\hline Negative & 67 & 64 \\
\hline \multicolumn{3}{|l|}{ Her2 expression } \\
\hline Positive & 51 & 49 \\
\hline Negative & 53 & 51 \\
\hline \multicolumn{3}{|l|}{ P53 expression } \\
\hline Positive & 73 & 70 \\
\hline Negative & 31 & 30 \\
\hline
\end{tabular}

dehydrated in ascending concentrations of alcohol (80-100\%). After xylol treatment, slides were covered.

Two investigators evaluated each stained section independently without knowing any clinical information. The proportions of positive cells were ranged from 10 to $100 \%$, while the intensity of staining was scored as 0 (negative), 1 (weak), 2 (moderate), and 3 (intense) in the most strongly stained tumor area. The immunoreactivity 
score for each case was taken as percentage of positive cells multiplied by the intensity of staining.

\section{RNA interference and transfection}

Fli-1-specific siRNAs (No. 1 and No. 2) were from Invitrogen. The target sequences were 5'-GGGAAAGUUC ACUGUUGGCCUAUAA-3' and 5'-AGGAGUGGAUC AAUCAGCCAGU-GAG-3', respectively. The target sequence of control siRNA against photinus pyralis luciferase gene (Invitrogen, CA) was 5'-GGAUUUCGAGUCG UCUUAAUGUAUA-3'. RNAiMAX transfection reagent was used for transient transfection following manufacturer's protocol (Invitrogen, CA).

\section{Cell proliferation assay}

SKOV3 cells were maintained in DMEM containing 1\% penicillin and streptomycin, supplemented with $10 \%$ fetal bovine serum (FBS), then incubated overnight at $37^{\circ} \mathrm{C}, 5 \% \mathrm{CO}_{2}$ with density $10 \%$ per well. The number of cell proliferation was measured by Trypan-blue exclusion assay from day 1 to day 4 .

\section{Cell migration and invasion assay}

Cell migration and invasion assays were carried out using Transwell (Corning Costar Corp, MA) membrane filter inserted in 24-well tissue culture plates $(6.5-\mathrm{mm}$ diameter, $8-\mu \mathrm{m}$ pore size). For migration assay, cells $\left(4 \times 10^{4}\right)$ suspended in serum-free medium were seeded on the upper chamber of transwell filters. Serum-containing medium was added to the lower chamber and incubated for $16 \mathrm{~h}$ at $37^{\circ} \mathrm{C}$. Nonmigrating cells were removed by wiping the upper side of the filter, and the remaining cells on the lower surface of the filter were fixed with $4 \%$ formaldehyde, stained with crystal purple and counted under a microscopy. Each determination represents the average of three individual experiments.

\section{Immunoblotting and antibodies}

Cells were lysed with radioimmunoprecipitation assay buffer (1\% Nonidet P-40, $50 \mathrm{mM}$ Tris-HCl, pH 7.4, $150 \mathrm{mM} \mathrm{NaCl}, 1 \%$ sodium deoxycholate, $0.1 \%$ SDS, plus protease inhibitor cocktail and $1 \mathrm{mM}$ phenylmethylsulfonyl fluoride). Proteins were separated by SDS-PAGE and analyzed by Western blotting. Antibodies to Fli-1 and $\beta$-actin were obtained from Santa Cruz Biotechnology (Santa Cruz Biotechnology, CA, USA).

\section{Cytoplasmic and nuclear fractionation}

Cells were harvested by trypsin-EDTA, collected by centrifugation and washed two times in ice-cold PBS. Pellets were lysed in buffer A containing 10 mM HEPES, pH 7.9, $10 \mathrm{mM} \mathrm{KCl}, 0.1 \mathrm{mM}$ EDTA, $0.1 \mathrm{mM}$ EDTA, $1 \mathrm{mM}$ PMSF, $1 \mathrm{mM}$ DTT, $1 \mathrm{mM} \mathrm{Na} 3 \mathrm{VO} 4$ supplemented with protease inhibitors and incubated for $15 \mathrm{~min}$ on ice. Thereafter,
NP-40 was added at a final concentration of $10 \%$ and lysates were oscillated. Nuclei were pelleted by centrifugation at $1000 \mathrm{~g}$ for $1 \mathrm{~min}$ at $4^{\circ} \mathrm{C}$ and supernatant containing cytoplasmic proteins $(\mathrm{C})$. The nucleic pellets were lysed in buffer B containing $20 \mathrm{mM}$ HEPES, pH 7.9, 0.4 M NaCl, 1 mM EDTA, 1 mM EGTA, 1 mM PMSF, $1 \mathrm{mM}$ Na3VO4, $1 \mathrm{mM}$ DTT, supplemented with protease inhibitors by repeated freezing and oscillating. Supernatants containing soluble nucleic proteins (N) were collected by centrifugation at $12000 \mathrm{~g}$ for $10 \mathrm{~min}$.

\section{Statistical analysis}

Statistical analysis was performed by using univariate (nonparametric rank sum test) and multivariate (logistic regression) analysis to evaluate the relationship between gene expression and clinicopathological parameters, including age, FIGO stage, histological grade, lymph nodal involvement, residual tumor size, CA125, ER expression, PR expression and P53 expression. Disease Free Survival (DFS) and Overall Survival (OS) were calculated by using the Kaplan-Meier method, and the differences were assessed by using the log-rank test. Comparison was made of groups with high Fli-1 expression (score $>$ median score) and low Fli-1 expression (score $\leq$ median score). The nonparametric rank sum test was used to determine the significance of the difference in the distribution of gene expression in cancer, borderline and normal samples. These analyses were performed by SPSS 13.0 Statistical Software. $P \leq 0.05$ was considered as statistically significant.

\section{Result}

Oncogenic Fli-1 is up-regulated in ovarian cancer tissues Immunohistochemical staining revealed that Fli-1 was generally expressed in the cytoplasm of ovarian cancer cells with various intensities (Figure 1). In primitive neuroectodermal tumor (PNET), Fli-1 was positive in nuclear (Figure 1f). Of the 104 EOC specimens examined in this study, Fli-1 was positive in $77(74 \%)$ cases. The scores of intensity were also analyzed. Eight (7.7\%) cases lacked Fli-1 expression; 19 (18.3\%) demonstrated weak expression of Fli-1; 60 (57.7\%) demonstrated moderate expression of Fli-1 and 17 (16.34\%) demonstrated a strong signal. Compared to EOC tissues, Fli-1 expression was either negative or expressed at negligible amount in normal ovaries and fallopian tube tissues. The expression levels of Fli-1 was significantly up-regulated in EOC tissues compared with normal ovarian $\left(p=4.56 \times 10^{-5}\right)$ and fallopian tube tissues $\left(p=8.25 \times 10^{-6}\right)$.

\section{Fli-1 expression is associated with clinicopathological characteristics of patients with EOC}

The expression rates of Fli-1 were 50.0\%, 62.4\%, 84\% and $83.3 \%$ in stage I, II, III and IV, respectively. There was no difference in the Fli- 1 scores of expression level 


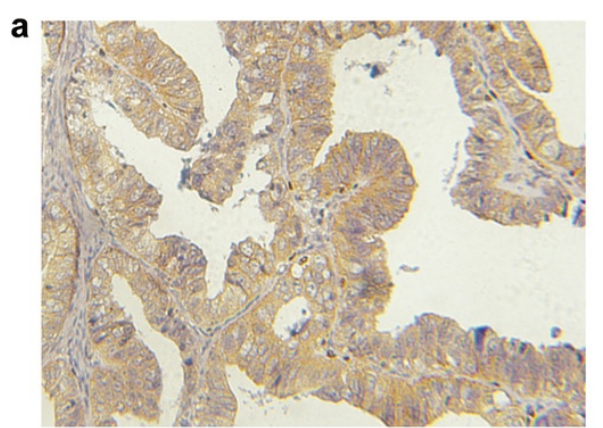

C

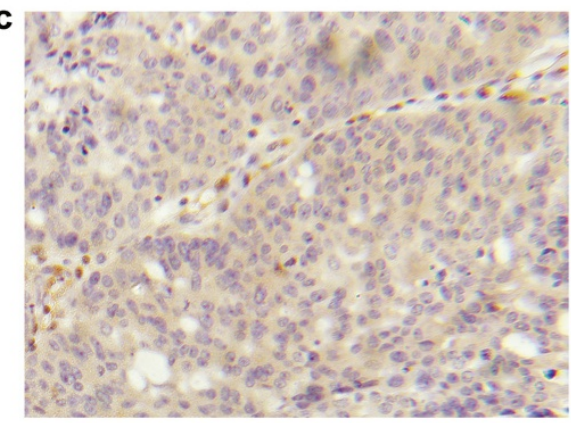

e

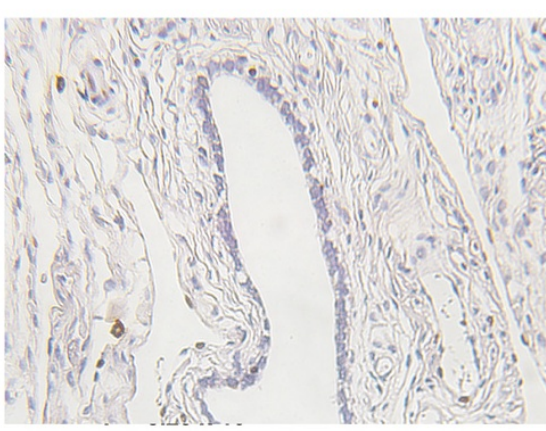

g

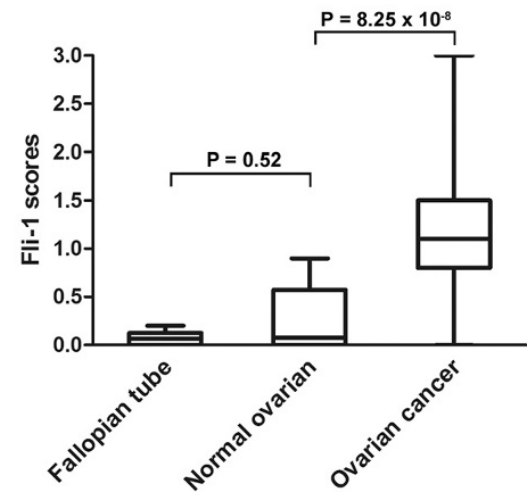

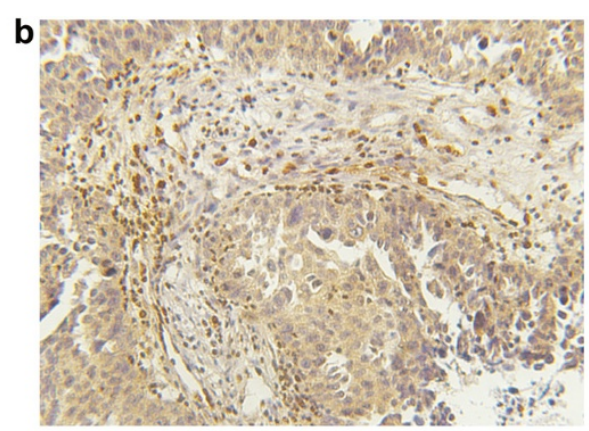
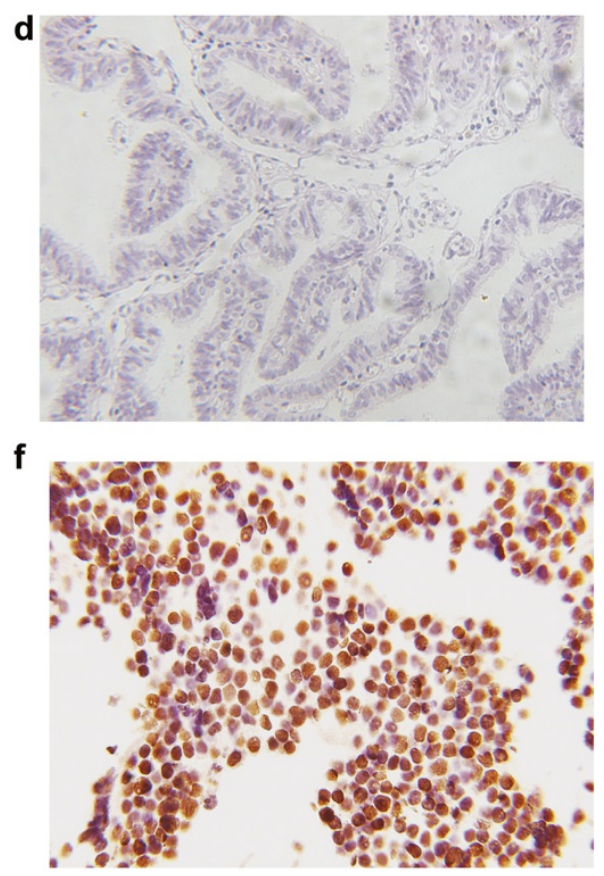


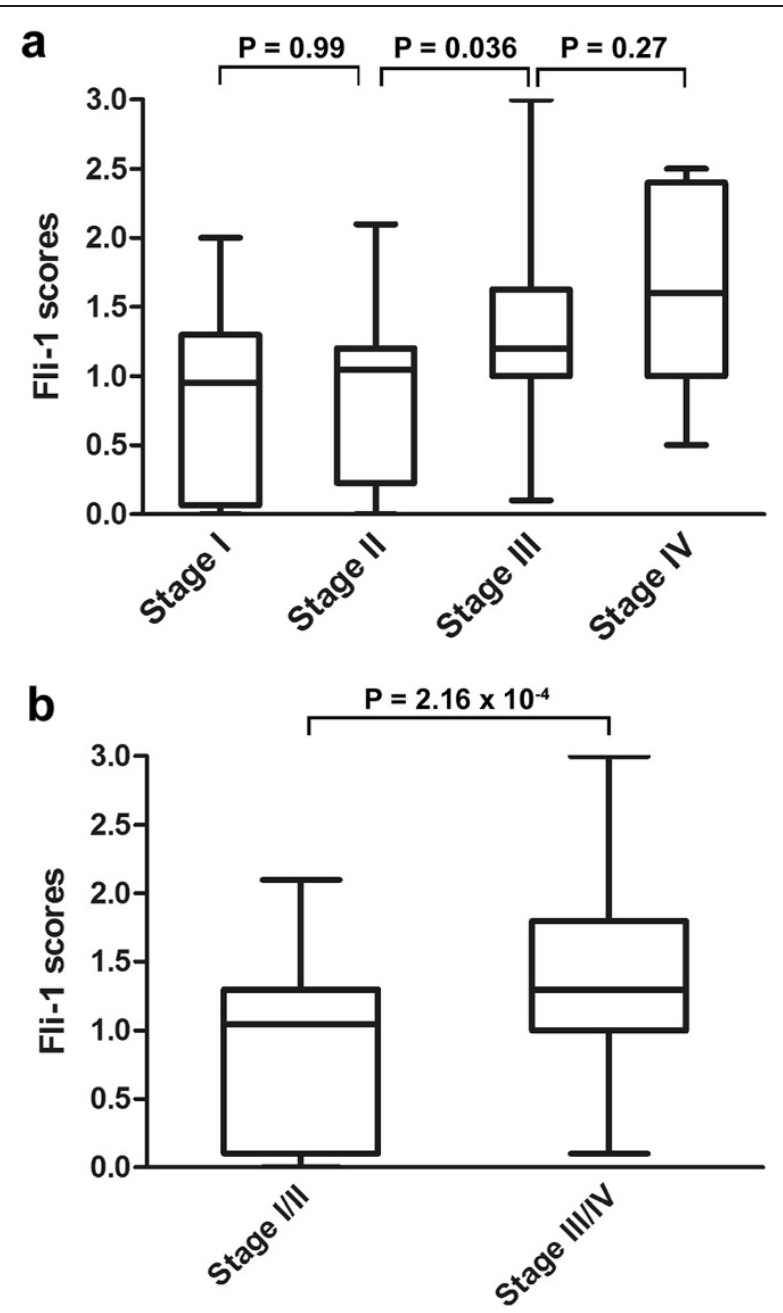

Figure 2 Inverse correlation between Fli-1 expression and tumor stage. (a) Box plot of Fli-1 expression in tumors with different stage. (b) Box plot of Fli-1 expression in low stage and advanced stage.

The relationship between Fli-1 expression and clinicopathologic parameters analyzed by univariate and multivariate analysis was illustrated in Table 2 . The expression of Fli-1 was significantly increased in the group of FIGO stage III and IV, lymph nodal involvement, and CA125 serum level $>35 \mathrm{U} / \mathrm{ml}(\mathrm{p}<0.05)$. However, Fli-1 expression was not correlated with age, histological grade, residual tumor size, and the expression of ER, PR, Her1, Her2 and P53 ( $p>0.05)$.

Fli-1 is a potential prognostic biomarker for ovarian cancer survival

The median follow-up interval was 32.8 months. Five patients were lost during follow-up. In univariate survival analyses for OS and DFS, 99 EOC patients were divided into two groups based on Fli-1 expression score in tumors, representing low (scores 0-1.1) and high (scores $>1.1$ ) expression of Fli-1. The Kaplan Meier survival curve in Figure 3 confirmed that patients with low expression of Fli-1 had better OS $(p=0.030)$ and DFS $(p=0.042)$. The median OS for the high Fli-1 expression group (48 patients, 33 events) was 27 month; however, the low Fli-1 expression group had significantly longer survival (48 months) (51 patients, 25 events). The median DFS was 23 month for the high Fli-1 expression group (48 patients, 33 events) but 43 month for the low Fli-1 expression group (51 patients, 26 events).

\section{Knockdown of Fli-1 inhibits cell proliferation in SKOV3 cells}

The cellular localization of Fli-1 was further examined in SKOV3 cells. The fractionation was verified by the presence of Lamin A/C in nuclei and tubulin in cytoplasm, and Fli-1 was present in the cytoplasm (Figure 4a).

Fli-1 was knocked down with target siRNA sequences in SKOV3 cells and the efficiency was detected by Western blotting (Figure 4b). Initial microscopic observation and cell counting with Trypan blue showed that the 
Table 2 Association between Fli-1 expression and clinicopathological parameters

\begin{tabular}{|c|c|c|c|c|}
\hline Parameter & p-value (uni) & p-value (multi) & $95 \% \mathrm{Cl}$ & OR \\
\hline Age at diagnosis & 0.848 & 0.485 & $0.70-2.13$ & 1.22 \\
\hline \multicolumn{5}{|l|}{$<50$ vs $\geq 50$} \\
\hline FIGO stage & $<0.010$ & $<0.010$ & $0.12-0.52$ & 0.25 \\
\hline \multicolumn{5}{|l|}{$\leq \|$ vs $>\|$} \\
\hline Histological grade & 0.309 & 0.153 & $0.18-1.31$ & 0.48 \\
\hline \multicolumn{5}{|l|}{ G1 vs G2\&G3 } \\
\hline Lymph nodal involvement & 0.015 & $<0.010$ & $0.23-0.80$ & 0.43 \\
\hline \multicolumn{5}{|l|}{ Positive vs negative } \\
\hline CA125 serum level & 0.006 & 0.013 & $0.04-0.68$ & 0.16 \\
\hline \multicolumn{5}{|l|}{$\leq 35 \mathrm{U} / \mathrm{ml} \mathrm{vs}>35 \mathrm{U} / \mathrm{ml}$} \\
\hline Residual tumor size & 0.341 & 0.421 & $0.41-1.45$ & 0.77 \\
\hline \multicolumn{5}{|l|}{$0 \mathrm{~mm}$ vs $0-10 \mathrm{~mm}$} \\
\hline ER expression & 0.575 & 0.425 & $0.46-1.39$ & 0.80 \\
\hline \multicolumn{5}{|l|}{ Positive vs negative } \\
\hline PR expression & 0.875 & 0.872 & $0.60-1.82$ & 1.05 \\
\hline \multicolumn{5}{|l|}{ Positive vs negative } \\
\hline Her1 expression & 0.228 & 0.230 & $0.80-2.58$ & 1.43 \\
\hline \multicolumn{5}{|l|}{ Positive vs negative } \\
\hline Her2 expression & 0.747 & 0.518 & $0.69-2.08$ & 1.20 \\
\hline \multicolumn{5}{|l|}{ Positive vs negative } \\
\hline P53 expression & 0.586 & 0.836 & $0.59-1.94$ & 1.07 \\
\hline Positive vs negative & & & & \\
\hline
\end{tabular}

proliferation of the cells treated with Fli-1 siRNA was significantly reduced (Figure $4 d, p<0.01$ ). The capability of migration of SKOV3 cells treated with control siRNA or Fli-1 siRNA were also examined. As shown in Figure 4c, knocking-down Fli-1 expression, however, did not have impact on the migration capacity $(p>0.05)$.

\section{Discussion}

EOC is a very aggressive gynecological tumor. Despite the use of multimodal therapy, their prognosis remains poor, with the probability of 5 years survival less than $30 \%$ for those presenting with advanced disease [24-26]. The molecular mechanisms involved in EOC remain largely unknown, and neither was the prediction biomarker for prognosis.

The present study is dedicated to identify biomarkers for prediction and intervention in the tumorigenesis and development of EOC. To study the association between Fli-1 and EOC, the expression of Fli-1 in EOC was detected by immunohistochemistry. Approximately $90 \%$ of ES/PNET had a specific $t(11 ; 22)(q 24 ; q 12)$ that results in fusion of the EWS and FLI-1 genes, and overexpression of FLI-1 protein. Therefore, PNET was used as positive control. The expression of Fli-1 in PNET was located in the nucleus. In contrast, we found that Fli-1 was predominantly located in the cytoplasm in $74 \%$ cases with various intensities. In recent years, with the full realization of the genesis for ovarian cancer, it is strongly suggested that high grade ovarian cancer originates not from the surface of the ovary, but from the epithelial layer of the neighboring fallopian tube epithelium $[27,28]$. Therefore, fallopian tube tissues were taken for control group together with normal ovaries.

The Fli-1 expression was negative in control group, but increased in early-stage tumors, and reached the highest level in advanced stage tumors. Clinicopathologic analysis of Fli-1 expression revealed that the high expression of Fli-1 was positively correlated with advanced tumor stage and positive lymph nodal involvement. This progressively increased expression profile paralleled with deterioration of the disease, suggested a role of Fli-1 in progression of EOC. Although it was shown no significant association between Fli-1 expression and histological grade, the imbalance in sample size between low grade $(G 1,10)$ and high grade (G2 and G3, 94) should be considered. At the same time, the study showed that high expression of biomarker CA125 was related to the staining of Fli-1, and the significance needed to be investigated.

The relationship between Fli-1 expression and prognosis was further analyzed by OS and DFS. Patients with 

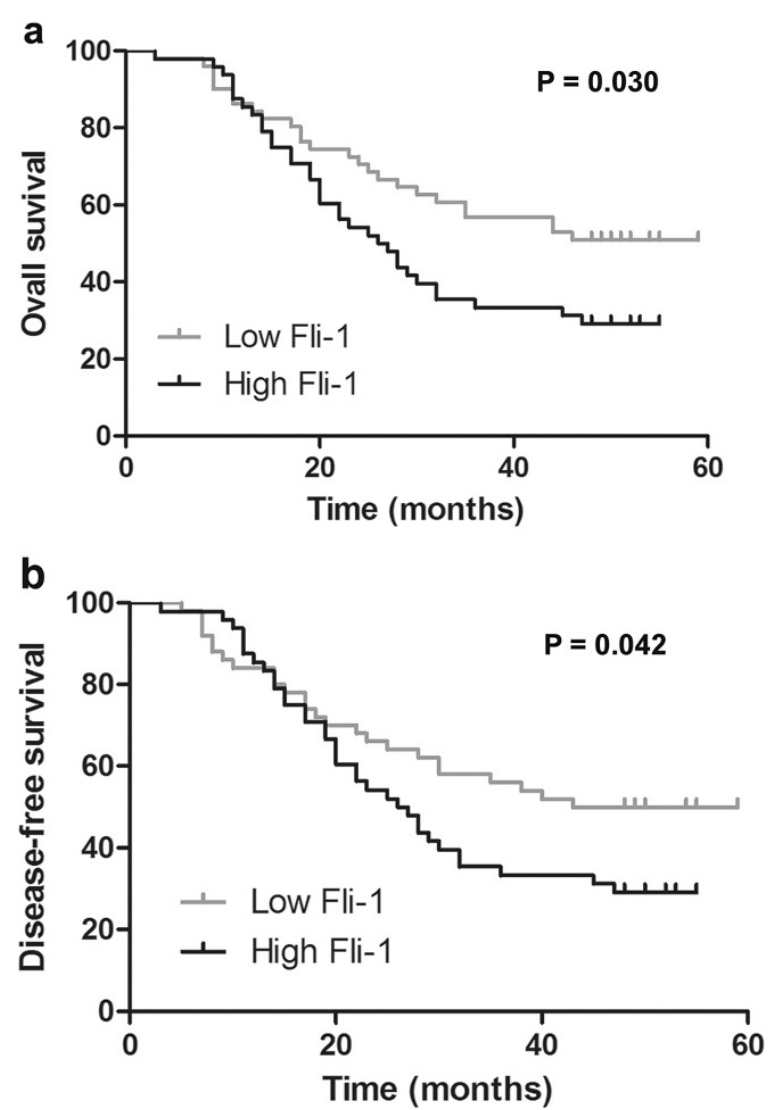

Figure 3 Fli-1 predicts clinical outcome of ovarian cancer. $(\mathbf{a}, \mathbf{b})$ Kaplan-Meier estimates of overall survival (a) and disease-free survival (b) in $99 \mathrm{EOC}$ patients. $P$ value refers to two-sided log-rank tests.

high expression of Fli-1 had poor OS and DFS, suggesting that Fli-1 is an attractive candidate for risk prognostication and the target therapy of EOC. As the treatment would have impact on survival, we also analyzed the treatment in the two groups. In this study, all of the patients were treated with standard regimens. Therefore, Fli-1 expression is highly associated with the survival in the patients with ovary cancer.

Increasing expression of Fli-1 is one of the common scenarios during tumor development and may be associated with the disease malignancy. To further study the role of Fli-1 overexpression in growth and metastasis, the function of Fli-1 in cell line was investigated. Functionally, we found knocking-down of Fli-1 reduced ovarian cancer cell proliferation, but did not affect tumor metastasis.

The expression of Fli-1 was predominantly found in the nuclei of Ewing sarcoma and leukemia [29]. In the present study, Fli-1 expression was mainly found in the cytoplasm of ovarian cancer tissues and SKOV3 cells. These data suggest that Fli-1 is required to function in the cytoplasm for ovarian cancer. Moreover, previous studies support that Fli-1 might function through protein-protein interaction or as being a transcription factor $[15,16,30,31]$. It was speculated that Fli-1 were widely expressed in various cancer tissues while it specifically played different roles. Thus, our results imply that Fli-1 may have distinct functions in signal transduction pathways in the cytoplasma, other than just being transcription factor. The status of Fli-1 in different cancers and the clinical implications of their expression during cancer development still need further investigation. In addition to the functional study, further investigation of the molecular mechanisms of Fli-1 is warranted.

Although invasive epithelial ovarian cancer is widely seen and treated as a single disease entity, there are different histological subtypes. Serous ovarian cancer studied in this study is the most common subtype. The expression status of Fli-1 in other subtypes also needs to be investigated in the future.

More importantly, we demonstrate a significant correlation between high Fli-1 immunoreactivity and shorter overall and disease-free survival. If high Fli-1 expression can be further confirmed to indicate poor prognosis, as suggested in this report, it may serve as an important prognostic marker and an attractive therapeutic target in 
b a
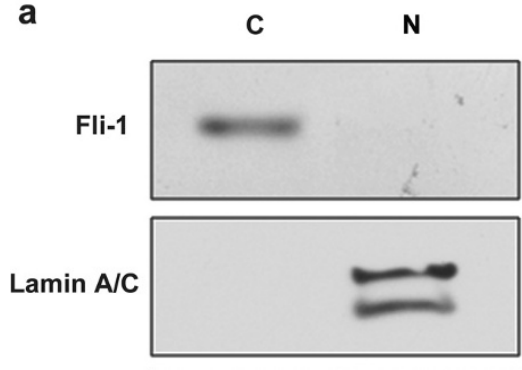

Tubulin

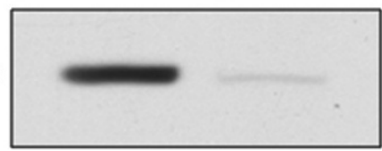

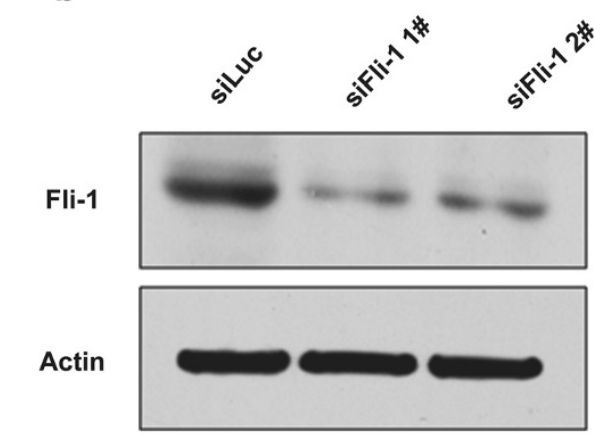

C

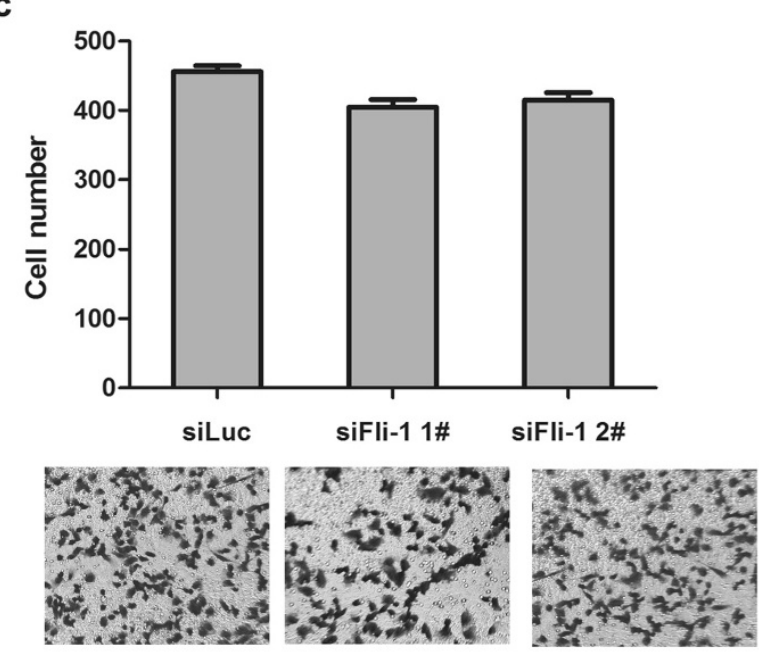

d

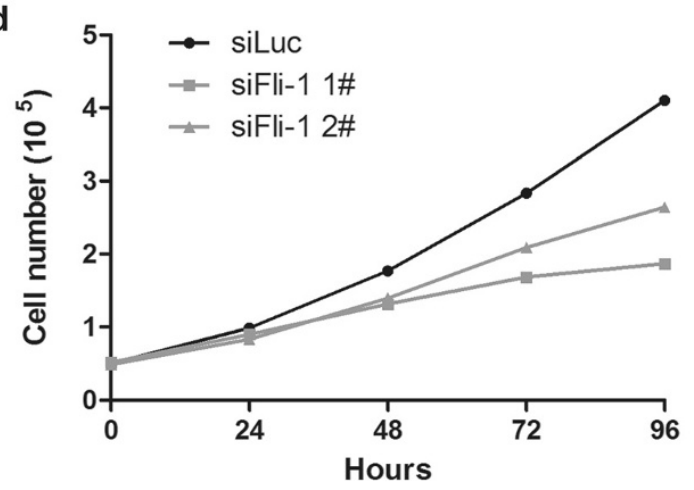

Figure 4 Present of Fli-1 and growth characteristics of SKOV3 cells with Fli-1 expression down-regulated. (a) Western blots showing the purity of the isolated nucleus/cytoplasm sample, nuclear (N) and cytoplasmic (C). (b) SiRNA transfection efficiency in tumor cells was measured by Western blotting; (c) Transwell migration assay of the indicated cell lines transfected with Fli-1 constructs or transient transfected with two different Fli-1 siRNA target sequence. (d) The growth curve displays the absolute counts of cells cultured in twelve-well plates during the 4-day treatment.

ovarian cancer. However, this study has limitation in sample size, and it is a retrospective and monocentric study. Therefore, further larger, multicentric studies are needed.

\section{Conclusion}

In conclusion, our findings suggest that Fli-1 is an important molecular change significantly related to tumorigenesis and progression of EOC. However, a larger 
cohort of patients with ovarian cancer and other cancer types is still required to further define the clinical significance of Fli-1 and its prognostic value in ovarian cancers in the future.

\section{Abbreviations}

EOC: Epithelial ovarian cancer; Fli-1: Friend leukemia virus integration 1; IHC: Immunohistochemical; FIGO: Federation of Gynecology and Obstetrics; OR: Odd ratio; DFS: Disease free survival; OS: Overall survival.

\section{Competing interests}

The authors declared that they have no financial or non-financial competing interests.

\section{Authors' contributions}

WS and LYH designed experiments, carried out the laboratory experiments, analyzed the data, interpreted the results and wrote the paper. WL and GJW participated in the design of the study and discussed analyses. YL and LY performed cell culture and transfection. ALL and JWC contributed the conception and design of this study, and helped to draft the manuscript. All authors read and approved the final manuscript.

\section{Acknowledgement}

This work was supported in part by grants from Ministry of Education Key Project of Science and Technology (311015) and National Natural Science Foundation of China for young scholars (30901702).

\section{Author details}

${ }^{1}$ Cancer center, the First Hospital of Jilin University, 71 Xinmin Street, Changchun 130021, China. ${ }^{2}$ Obstetrics and Gynecology, the General Hospital of Chinese People's Liberation Army, Beijing, China. ${ }^{3}$ Institute of Basic Medical Sciences, National Center of Biomedical Analysis, 27 Tai-Ping Road, Beijing 100850, China.

Received: 21 September 2013 Accepted: 29 May 2014

Published: 12 June 2014

\section{References}

1. Jemal A, Bray F, Center MM, Ferlay J, Ward E, Forman D: Global cancer statistics. CA Cancer J Clin 2011, 61:69-90.

2. Siegel R, Naishadham D, Jemal A: Cancer statistics. CA Cancer J Clin 2012, 62:10-29.

3. Onda T, Yoshikawa H: Neoadjuvant chemotherapy for advanced ovarian cancer: overview of outcomes and unanswered questions. Expert Rev Anticancer Ther 2011, 11:1053-1067.

4. Kurman RJ, Visvanathan K, Roden R, Wu TC, Shih IM: Early detection and treatment of ovarian cancer: shifting from early stage to minimal volume of disease based on a new model of carcinogenesis. Am J Obstet Gynecol 2008, 198:351-356.

5. Ben-David Y, Bernstein A: Friend virus-induced erythroleukemia and the multistage nature of cancer. Cell 1991, 66:831-834.

6. Klemsz MJ, Maki RA, Moore J, Hromas R: Characterization of the ets oncogene family member, fli-1. J Biol Chem 1994, 268:5769-5773.

7. Maroulakou IG, Bowe DB: Expression and function of Ets transcription factors in mammalian development: a regulatory network. Oncogene 2000, 19:6432-6442.

8. Oikawa T, Yamada T: Molecular biology of the Ets family of transcription factors. Gene 2003, 303:11-34.

9. Davidson B, Reich R, Goldberg I, Gotlieb WH, Kopolovic J, Berner A, Ben-Baruch G, Bryne M, Nesland JM: Ets-1 messenger RNA expression is a novel marker of poor survival in ovarian carcinoma. Clin Cancer Res 2001, 7:551-557.

10. Oikawa T: ETS transcription factors: possible targets for cancer therapy. Cancer Sci 2004, 95:626-633.

11. Melet F, Motro B, Rossi DJ, Zhang L, Bernstein A: Generation of a nove Fli-1 protein by gene targeting leads to a defect in thymus development and a delay in Friend virus-induced erythroleukemia. Mol Cell Biol 1996, 16:2708-2718.

12. Truong AH, Ben-David $Y$ : The role of Fli-1 in normal cell function and malignant transformation. Oncogene 2000, 19:6482-6489.
13. Spyropoulos DD, Pharr PN, Lavenburg KR, Jackers P, Papas TS, Ogawa M, Watson DK: Hemorrhage, impaired hematopoiesis, and lethality in mouse embryos carrying a targeted disruption of the Fli1 transcription factor. Mol Cell Biol 2000, 20:5643-5652.

14. Liu F, Walmsley M, Rodaway A, Patient R: Fli1 acts at the top of the transcriptional network driving blood and endothelial development. Curr Biol 2008, 18:1234-1240.

15. Lakhanpal GK, Vecchiarelli-Federico LM, Li YJ, Cui JW, Bailey ML, Spaner DE, Dumont DJ, Barber DL, Ben-David Y: The inositol phosphatase SHIP-1 is negatively regulated by Fli-1 and its loss accelerates leukemogenesis. Blood 2010, 11:6428-6436.

16. Cui JW, Vecchiarelli-Federico LM, Li YJ, Wang GJ, Ben-David Y: Continuous Fli-1 expression plays an essential role in the proliferation and survival of F-MuLV-induced erythroleukemia and human erythroleukemia. Leukemia 2009, 23:1311-1319.

17. Erkizan HV, Uversky VN, Toretsky JA: Oncogenic partnerships: EWS-FLI1 protein interactions initiate key pathways of Ewing's sarcoma. Clin Cancer Res 2010, 16:4077-4083.

18. Takigami I, Ohno T, Kitade Y, Hara A, Nagano1 A, Kawai G, Saitou M, Matsuhashi A, Yamada K, Shimizu K: Synthetic siRNA targeting the breakpoint of EWS/Fli-1 inhibits growth of Ewing sarcoma xenografts in a mouse model. Int J Cancer 2011, 128:216-226.

19. Zhang JJ, Guo HY, Zhang H, Wang HB, Qian GX XF, Hoffman AR, Hu JF, Ge S: Putative tumor suppressor miR-145 inhibits colon cancer cell growth by targeting oncogene Friend leukemia virus integration 1. Cancer 2011, 117:86-95.

20. Tamir A, Howard J, Higgins RR, Li YJ, Berger L, Zacksenhaus E, Reis M, Ben-David Y: Fli-1, an Ets-related transcription factor, regulates erythropoietin- induced erythroid proliferation and differentiation: evidence for direct transcriptional repression of the $\mathrm{Rb}$ gene during differentiation. Mol Cell Biol 1999, 19:4452-4464

21. Yi H, Fujimura Y, Ouchida M, Prasad DD, Rao VN, Reddy ES: Inhibition of apoptosis by normal and aberrant Fli-1 and erg proteins involved in human solid tumors and leukemias. Oncogene 1997, 14:1259-1268.

22. Pereira R, Quang CT, Lesault I, Dolznig H, Beug H, Ghysdael J: FLI-1 inhibits differentiation and induces proliferation of primary erythroblasts. Oncogene 1999, 18:1597-1608.

23. Li YJ, Zhao X, Vecchiarelli-Federico LM, Li Y, Datti A, Cheng Y, Ben-David Y: Drug-mediated inhibition of Fli-1 for the treatment of leukemia. Blood Canc J 2012, 2:e54.

24. Jemal A, Siegel R, Ward E, Hao Y, Xu J, Thun MJ: Cancer statistics, 2009. CA Cancer J Clin 2009, 59:225-249.

25. Goff BA, Mandel L, Muntz HG, Melancon $\mathrm{CH}$ : Ovarian carcinoma diagnosis. Cancer 2000, 89:2068-2075.

26. Ozols RF: Treatment goals in ovarian cancer. Int J Gynecol Cancer 2005, 15:S3-S11.

27. Kessler M, Fotopoulou C, Meyer T: The molecular fingerprint of high grade serous ovarian cancer reflects its fallopian tube origin. Int J Mol Sci 2013, 14:6571-6596.

28. Levanon K, Crum C, Drapkin R: Newinsights into the pathogenesis of serous ovarian cancer and its clinical impact. J Clin Oncol 2008, 26:5284-5293.

29. Bujor AM, Asano Y, Haines P, Lafyatis R, Trojanowska M: The C-ABL tyrosine kinase controls PKC $\delta$ induced Fli1 phosphorylation in human dermal fibroblasts. Arthritis Rheum 2011, 63:1729-1737.

30. Eisbacher M, Holmes ML, Newton A, Hogg PJ, Khachigian LM, Crossley M, Chong BH: Protein-protein interaction between Fli-1 and GATA-1 mediates synergistic expression of megakaryocyte-specific genes through cooperative DNA binding. Mol Cell Biol 2003, 23:3427-3441.

31. Starck J, Cohet N, Gonnet C, Sarrazin S, Doubeikovskaia Z, Doubeikovski A, Verger A, Duterque-Coquillaud M, Morle F: Functional cross-antagonism between transcription factors FLI-1 and EKLF. Mol Cell Biol 2003, 23:1390-1402.

doi:10.1186/1471-2407-14-424

Cite this article as: Song et al:: Oncogenic Fli- 1 is a potential prognostic marker for the progression of epithelial ovarian cancer. BMC Cancer 2014 14:424. 